\title{
The diffusion of radiation in moving media
}

\section{Stochastic representation of spectral lines}

\author{
R. Wehrse ${ }^{1,3}$, B. Baschek ${ }^{1}$, and W. von Waldenfels ${ }^{2,3}$ \\ 1 Institut für Theoretische Astrophysik, Tiergartenstrasse 15, 69121 Heidelberg, Germany \\ 2 Institut für Angewandte Mathematik, Im Neuenheimer Feld 294, 69120 Heidelberg, Germany \\ 3 Interdisziplinäres Zentrum für Wissenschaftliches Rechnen, Im Neuenheimer Feld 368, 69120 Heidelberg, Germany
}

Received 11 January 2002 / Accepted 15 May 2002

\begin{abstract}
In this paper we present analytical expressions for the radiative flux, the effective extinction coefficient, and the radiative acceleration deep inside a differentially moving, very optically thick medium with many spectral lines. It is shown that the line contribution is essentially given by the characteristic function of wavelength averages of the extinction coefficient. It can be calculated either by means of the generalized opacity distribution function or by means of a Poisson point process model. Several examples are given to demonstrate the basic consequences of line densities, widths, and strengths, as well as velocity gradients on the diffusive flux. We demonstrate that for Poisson distributed lines the diffusive flux decreases with increasing absolute values of the velocity gradient (i.e. that the flux has a maximum for static media), and that such lines have hardly a direct influence on the radiative acceleration.
\end{abstract}

Key words. diffusion - radiative transfer - stars: interiors - novae, cataclysmic variables - supernovae: general

\section{Introduction}

This is the third paper in a series in which the effects of differential motions on the radiative quantities in optically thick media are discussed. In the first paper (Wehrse et al. 2000a, Paper I) the general expression for the flux was derived from the analytical solution of the transfer equation for moving media (Baschek et al. 1997). In the second paper (Wehrse et al. 2000b, Paper II) the limits of small and large velocity gradients $w$ were considered. For the important case of small gradients it was shown that the mean free path and its (logarithmic) wavelength derivatives are the dominant quantities (as for the Rosseland mean in the static case) and that in this case isolated symmetric spectral lines contribute negatively in second order in $w$ to the flux, i.e. the lines always lead to a reduction of the flux. Several examples demonstrated that various parts of a lines profile influence the flux in very different ways (even changes of sign occur!) and that this leads to a very intricate behavior when lines overlap.

In fact, in astrophysical applications one frequently has to deal with a very large number of spectral lines that more or less overlap. In such a case it seems appropriate not to consider each line individually but to use a stochastic approach since this is much faster and easier to employ in radiation-hydrodynamic modelling and the loss in accuracy seems to be minimal.

Send offprint requests to: $\mathrm{R}$. Wehrse,

e-mail: wehrse@ita.uni-heidelberg.de
In addition, the stochastic approach allows us to study the various intricate interdependencies.

As has been shown by Baschek et al. (2001), there are (at least) two simple ways for including spectral lines statistically in the modelling of radiation fields for static and differentially moving media: the generalization of the well known opacity distribution function and the concept of a Poisson point process (see also Wehrse et al. 1998). Both approaches are not completely independent since e.g. the generalized opacity distribution functions can be obtained from the characteristic function of the Poisson point process by means of a Fourier transform.

In this paper we combine the concepts of Baschek et al. with those of Paper I in order to obtain convenient expressions for the flux, the corresponding effective extinction, and the radiative acceleration in optically thick media with many overlapping lines. In Sect. 2 we first briefly review the basics of the generalized opacity function and the expectation values of the Poisson point process which are relevant for the diffusion of radiation. Then, in the following two sections, the expressions for the radiative flux (Sect. 3) and the radiative acceleration (Sect. 4) - in the diffusion limit - are derived for the stochastic presentation of the spectral lines by opacity distribution functions and by the Poisson point process. The limits of these expressions for a vanishing velocity gradient $w$, i.e. for the static case, may serve as reference terms for the discussion of the effects due to the motions. In Sects. 5 and 6 it is shown that the limiting cases of small and large $w$ yield noticeably simpler 
expressions for the radiative expressions. Section 7 is devoted to several examples which have been chosen to demonstrate the different behaviors of continua and lines and to indicate how the line densities, strengths etc. influence the monochromatic flux or, equivalently, the effective extinction. Expressions for the total flux, obtained by integration over the wavelength, are given in Sect. 8. The central question, in which way the flux, calculated upon the assumption of Poisson distributed lines, is changed when a velocity field is added to a static medium is discussed in Sect. 9. We conclude the paper in Sect. 10 with a discussion and outlook.

In this paper, equations of the first two papers of this series are cited by (I:n) and (II:m), respectively.

\section{Stochastic line extinction}

We start with the (deterministic) monochromatic extinction coefficient (II:8)

$\chi(\xi)=\chi_{\mathrm{c}}(\xi)+\sum_{l=1}^{L} \chi_{l}(\xi)=\chi_{\mathrm{c}}(\xi)+X(\xi)$

where $\chi_{\mathrm{c}}(\xi)$ is the continuous extinction coefficient, $X(\xi)$ the contribution of $L$ of spectral lines (each one labelled by $l$ ), and $\xi=\ln \lambda$ a logarithmic wavelength scale. The line extinction coefficient is written as

$\chi_{l}(\xi)=A_{l} \cdot \Phi_{l}\left(\xi, \gamma_{l}\right)$

with $\Phi_{l}$ being the profile function with the intrinsic width $\gamma_{l}$, normalized by $\int_{\text {line }} \Phi_{l}\left(\xi, \gamma_{l}\right) \mathrm{d} \xi=1$, and $A_{l}$ the line strength.

Throughout this paper, it is assumed that $\chi_{\mathrm{c}}(\xi)$ does not vary significantly with $\xi$ over a small range of several line widths, but may change over larger portions of the spectrum so that terms involving $\chi_{\mathrm{c}}^{\prime}=\partial \chi_{\mathrm{c}} / \partial \xi$ can be neglected when small $\xi$-intervals are considered.

In the following, we first briefly recapitulate the basic concepts of the two methods which we will apply to differentially moving media using a stochastic description of a very large number $(L \gg 1)$ of spectral lines, namely of the opacity distribution functions and the presentation of the lines by a Poisson point process. For details, we refer to Baschek et al. (2001), Wehrse et al. (1998), and to textbooks on probability theory, e.g. Feller (1966) and Fisz (1978).

\subsection{Generalized opacity distribution function}

The conventional static opacity distribution function can be interpreted as the probability

$\mathbb{P}(X(\xi) \in(x, x+\mathrm{d} x))=p(x ; \xi) \mathrm{d} x$

that the contribution of all $L$ spectral lines to the monochromatic extinction coefficient $X=\sum_{l} \chi_{l}$ at any $\xi$ has a value that lies between $x$ and $x+\mathrm{d} x$. Note that the continuous extinction is not included in the opacity distribution function and is treated as deterministic function.

For a differentially moving medium the generalized opacity distribution function

$\tilde{\mathbb{P}}(\bar{X}(\xi ; \Delta) \in(\bar{x}, \bar{x}+\mathrm{d} \bar{x}))=\tilde{p}(\bar{x} ; \xi, \Delta) \mathrm{d} \bar{x}$, is defined as the probability that the mean line extinction coefficient,

$\bar{X}(\xi ; \Delta)=\frac{1}{\Delta} \int_{\xi-\Delta}^{\xi} \sum_{l=1}^{L} \chi_{l}(\zeta) \mathrm{d} \zeta=\frac{1}{\Delta} \int_{\xi-\Delta}^{\xi} X(\zeta) \mathrm{d} \zeta$,

over the logarithmic wavelength interval $(\xi-\Delta, \xi)$ has a value that lies between $\bar{x}$ and $\bar{x}+\mathrm{d} \bar{x}$. Note that $\lim _{\Delta \rightarrow 0} \bar{X}(\xi ; \Delta)=X(\xi)$. As probabilities, the distribution functions are normalized according to $\int_{0}^{\infty} p(x ; \xi) \mathrm{d} x=1$ and $\int_{0}^{\infty} \tilde{p}(\bar{x} ; \xi, \Delta) \mathrm{d} \bar{x}=1$, respectively.

The distribution function for the moving case depends on two parameters, $\xi$ and $\Delta$. This two-dimensional set of data can e.g. be calculated from a given deterministic line list in a way analogous to the well-known static case without any a priori knowledge of the velocity field.

\subsection{Poisson point process}

The assumption of a Poisson point process is a good and flexible approximation of a set of deterministic "real" line data with a very large number $L$ of lines (Wehrse et al. 1998). By this formalism, e.g. the expectation value of the radiative flux can be calculated largely analytically so that the effects of motions and various line ensembles can be well investigated whereas this is virtually impossible when a generalized opacity distribution function (see Sect. 2.1) is employed that has been determined numerically from a deterministic list.

In this paper we consider only singly-peaked, symmetric lines with differentiable normalizable profile functions (mathematically, of type $L_{1}$ ). However, most expressions are also valid for asymmetric lines which are strictly monotonic on either side.

In order to apply the Poisson point process, we introduce in (1) the wavelength position $\hat{\xi}_{l}$ of the line center - or in case of an asymmetric lines of the profile maximum - as an additional variable of $\chi$ besides the "normal" logarithmic wavelength scale $\xi$ and the multidimensional line parameter $\vartheta_{l}$ comprising the line strength $A_{l}$, the intrinsic line width $\gamma_{l}$, and the line type, i.e. $\vartheta_{l}=\left\{A_{l}, \gamma_{l}\right.$, line type $\}$. Here we assume that all spectral lines have the same type of profile $\Phi$ so that $\vartheta_{l}=\left\{A_{l}, \gamma_{l}\right\}$. The line extinction coefficient

$$
\begin{aligned}
X(\xi)=\sum_{l=1}^{L} \chi_{l}(\xi) & =\sum_{l=1}^{L} \chi\left(\hat{\xi}_{l}, \vartheta_{l}, \xi-\hat{\xi}_{l}\right) \\
& =\sum_{l=1}^{L} A_{l} \cdot \Phi\left(\hat{\xi}_{l}, \gamma_{l}, \xi-\hat{\xi}_{l}\right)
\end{aligned}
$$

is thus considered as a function of three variables. In view of the large number of lines, not only $\xi$ but also the line position and the line parameter are treated as continuous variables $\hat{\xi}$ and $\vartheta($ or $A, \gamma)$, i.e.

$\chi(\hat{\xi}, \vartheta, \xi-\hat{\xi})=A \cdot \Phi(\hat{\xi}, \gamma, \xi-\hat{\xi})$.

For the description of the spectral lines by a Poisson point process we need - in addition to $\chi(\hat{\xi}, \vartheta, \xi-\hat{\xi})-$ the distributions of the line positions and shape parameters. This information 
is contained in the mean density of lines, $\varrho(\hat{\xi}, \vartheta)$, with properties $\vartheta$ within the interval $\mathrm{d} \hat{\xi}$. Then the mean number of lines in the interval $\mathrm{d} \hat{\xi} \mathrm{d} \vartheta$ is $\varrho(\xi, \vartheta) \mathrm{d} \xi \mathrm{d} \vartheta$, and the mean number or the expectation value of all lines is

$\langle L\rangle=\int_{\Theta} \int_{-\infty}^{\infty} \varrho(\hat{\xi}, \vartheta) \mathrm{d} \hat{\xi} \mathrm{d} \vartheta$.

The total number $L$ of lines is now a random variable, but its expectation value $\langle L\rangle$ differs very little from the actual number of lines since $L$ is very large, i.e. $\langle L\rangle \simeq L$.

The approximation of the lines by a Poisson point process requires that there are no correlations between their positions. For the line positions we assume an equal-probability distribution; our algorithm allows us to choose the distribution of the parameter $\vartheta$ for the line properties more or less arbitrarily. Of course, for any specific astronomical object under consideration these parameters are determined by the particular chemical composition, the temperature, and the pressure etc.

In a Poisson point process the total number of lines is Poisson distributed with the probability

$\mathbb{P}\{L=n\}=\frac{\langle L\rangle^{n}}{n !} \mathrm{e}^{-\langle L\rangle}$

that the actual number of lines $L$ has the value $n$.

In the formalism of the Poisson point process, the expectation value of any radiative quantity is a functional of the two basic functions, the line extinction coefficient $\chi(\hat{\xi}, \vartheta, \xi-\hat{\xi})$ and the line density $\varrho(\hat{\xi}, \vartheta)$. For any actual calculation these basic functions have to be specified. The inclusion of the (deterministic) continuous extinction is trivial.

We point out that $\varrho(\hat{\xi}, \vartheta)$ has to be a slowly varying function, since it is usually determined by means of an ergodic hypothesis, i.e. $\varrho$ should not vary significantly over a few line widths.

\subsubsection{Important expectation values and nomenclature}

According to Wehrse et al. (1998) the typical expectation value - occurring in the expressions of the specific intensity and the flux - which describes the contribution of Poisson distributed lines is

$$
\begin{aligned}
\Omega(\xi, \xi-w s ; w)=\left\langle\mathrm{e}^{-\bar{X}(\xi ; w) \cdot s}\right\rangle \\
=\left\langle\exp \left(-\frac{1}{w} \sum_{l=1}^{L} \int_{\xi-w s}^{\xi} \chi\left(\hat{\xi}_{l}, \vartheta_{l}, \zeta-\hat{\xi}_{l}\right) \mathrm{d} \zeta\right)\right\rangle \\
=\exp \left(\int_{\Theta}^{\infty} \varrho \int_{-\infty}^{\infty} \varrho(\hat{\xi}, \vartheta)\right. \\
\left.\quad \times\left\{\exp \left(-\frac{1}{w} \int_{\xi-w s}^{\xi} \chi(\hat{\xi}, \vartheta, \zeta-\hat{\xi}) \mathrm{d} \zeta\right)-1\right\} \mathrm{d} \hat{\xi} \mathrm{d} \vartheta\right),
\end{aligned}
$$

where we have adopted the convenient abbreviation $\Omega$.

Its static limit $(w \rightarrow 0)$ is given by

$$
\begin{aligned}
\Omega(\xi, \xi ; 0) & =\left\langle\mathrm{e}^{-X(\xi) \cdot s}\right\rangle \\
& =\exp \left(\int_{\Theta} \int_{-\infty}^{\infty} \varrho(\hat{\xi}, \vartheta)\left\{\mathrm{e}^{-\chi(\hat{\xi}, \vartheta, \xi-\hat{\xi}) \cdot s}-1\right\} \mathrm{d} \hat{\xi} \mathrm{d} \vartheta\right) .
\end{aligned}
$$

The static expression may well serve as reference to describe the effects of the differential motions in the medium.

Shifting the $\zeta$ - and $\hat{\xi}$-scales by $w s / 2$, i.e. transforming $\zeta \rightarrow$ $\zeta+w s / 2$ and $\hat{\xi} \rightarrow \hat{\xi}+w s / 2$, leads to the symmetric form of Eq. (10),

$$
\begin{aligned}
& \Omega(\xi, \xi-w s ; w)=\exp \left(\int_{\Theta} \int_{-\infty}^{\infty} \varrho(\hat{\xi}, \vartheta)\right. \\
& \left.\quad \times\left\{\exp \left(-\frac{1}{|w|} \int_{\xi-|w| s / 2}^{\xi+|w| s / 2} \chi(\hat{\xi}, \vartheta, \zeta-\hat{\xi}) \mathrm{d} \zeta\right)-1\right\} \mathrm{d} \hat{\xi} \mathrm{d} \vartheta\right),
\end{aligned}
$$

which immediately shows that the expectation value of the flux does not depend on the sign or direction of the velocity gradient. This important result is a consequence of the assumption that the line centers of Poisson distributed lines follow an equal-probability distribution on the $\xi$ axis.

As a shorthand notation we will frequently use

$$
\begin{aligned}
\tilde{\mathcal{E}}(Y)= & \int_{\Theta} \int_{-\infty}^{\infty} \rho(\hat{\xi}, \vartheta) Y(\hat{\xi}, \vartheta, \xi-\hat{\xi}) \\
& \times \exp \left(-\frac{1}{w} \int_{\xi-w s}^{\xi} \chi(\hat{\xi}, \vartheta, \zeta-\hat{\xi}) \mathrm{d} \zeta\right) \mathrm{d} \hat{\xi} \mathrm{d} \vartheta
\end{aligned}
$$

where $Y=Y(\hat{\xi}, \vartheta, \xi-\hat{\xi})$. The abbreviation $\tilde{\mathcal{E}}$ may be considered as a particular kind of average of the line extinction coefficient over its parameters $\hat{\xi}$ and $\vartheta$ in the wavelength interval $w s$. Its static limit is

$$
\begin{aligned}
\mathcal{E}(Y) & =\lim _{w \rightarrow 0} \tilde{\mathcal{E}}(Y) \\
& =\int_{\Theta} \int_{-\infty}^{\infty} \varrho(\hat{\xi}, \vartheta) \cdot Y(\hat{\xi}, \vartheta, \xi-\hat{\xi}) \cdot \mathrm{e}^{-\chi(\hat{\xi}, \vartheta, \xi-\hat{\xi}) \cdot s} \mathrm{~d} \hat{\xi} \mathrm{d} \vartheta
\end{aligned}
$$

Since $\varrho$ does not vary over a few line widths, it is reasonable to take it out of the integral over $\hat{\xi}$ so that

$\mathcal{E}(Y)=\int_{\Theta} \varrho(\xi, \vartheta)\left[\int_{-\infty}^{\infty} Y \cdot \mathrm{e}^{-\chi \cdot s} \mathrm{~d} \hat{\xi}\right] \mathrm{d} \vartheta$

If furthermore a function $Z(\hat{\xi}, \vartheta, \xi-\hat{\xi})$ can be found so that $Y \mathrm{e}^{-\chi \cdot s}=\partial Z / \partial \hat{\xi}$ and $\lim _{w \rightarrow+\infty} Z=\lim _{w \rightarrow-\infty} Z$ holds, then $\mathcal{E}(Y)=0$.

\section{Radiative flux in the diffusion approximation}

The expression for the monochromatic radiative flux in the diffusion limit in the direction $\mathbf{s}=\boldsymbol{s} \boldsymbol{n}$ ( $\boldsymbol{n}$ : unit vector) at a (logarithmic) wavelength $\xi$ and at a position $s_{0}$ "deep inside" the medium, i.e. far from all surfaces, has been derived by Wehrse et al. (2000a, I:12, 13, 39, and 41) from the comoving radiative transfer equation. The flux vector

$$
\boldsymbol{F}_{w}\left(s_{0}, \xi\right)=\int_{2 \pi} \mathcal{F}\left(s_{0}, \xi ; w\right) \boldsymbol{n} \mathrm{d} \omega
$$

is obtained by integration of the flux-like antisymmetric average of the specific intensities in the direction $\boldsymbol{n}, \mathcal{F}\left(s_{0}, \xi ; w\right)$, over half of the sphere. For short, we subsequently call $\mathcal{F}$ the monochromatic flux. 
Since in this paper we apply a stochastic description, we have to evaluate its expectation value (cf. Wehrse et al. 1998)

$$
\begin{aligned}
\left\langle\mathcal{F}\left(s_{0}, \xi ; w\right)\right\rangle & =2 g\left(s_{0}, \xi\right) \int_{0}^{\infty}\left\langle\exp \left(-\frac{1}{w} \int_{\xi-w s}^{\xi} \chi(\zeta) \mathrm{d} \zeta\right)\right\rangle \mathrm{d} s \\
& =2 g\left(s_{0}, \xi\right) \int_{0}^{\infty} \mathrm{e}^{-\chi_{\mathrm{c}}(\xi) \cdot s}\left\langle\mathrm{e}^{-\bar{X}(\xi ; w s) \cdot s}\right\rangle \mathrm{d} s
\end{aligned}
$$

and its static limit $(w \rightarrow 0)$

$$
\left\langle\mathcal{F}\left(s_{0}, \xi\right)\right\rangle=2 g\left(s_{0}, \xi\right) \int_{0}^{\infty} \mathrm{e}^{-\chi_{\mathrm{c}}(\xi) \cdot s}\left\langle\mathrm{e}^{-X(\xi) \cdot s}\right\rangle \mathrm{d} s .
$$

Here $s$ is an integration variable for the geometrical depth. In this paper we consider all gradients such as that of the temperature $T$ or that of the velocity $\beta=v / c$ only in the direction $\boldsymbol{n}$, and hence drop the variable $\boldsymbol{n}$ in our notation. $g\left(s_{0}, \xi\right)=$ $(\partial B(T, \xi) / \partial T) \cdot(\partial T / \partial s)$ is the weighting function (I:6) with $B(T, \xi)$ being the Planck function, $w=\mathrm{d} \beta / \mathrm{d} s$ is the velocity gradient (I:33) which we assume to be independent of $s$, and $\chi(\xi)$ is the extinction coefficient according to Eq. (1). Note that the sign of $\mathcal{F}$ changes when the directions of $s$ or of the intensities are reversed.

In the diffusion approximation, $\chi$ is allowed to vary only slowly with $s_{0}$, i.e. it has to be nearly constant over one mean free path of the photons in the continuum. $\bar{X}(\xi, \Delta)$ is the average line extinction coefficient as defined by Eq. (5) in which $\Delta=w s$ depends on the velocity field.

Furthermore, we define the effective monochromatic extinction coefficient $\chi_{\text {eff }}(\xi ; w)$ in the direction $\boldsymbol{n}$ so that $\langle\mathcal{F}\rangle$ is expressed in a form resembling that of the static Rosseland mean:

$$
\left\langle\mathcal{F}\left(s_{0}, \xi ; w\right)\right\rangle=\frac{2 g\left(s_{0}, \xi\right)}{\chi_{\mathrm{eff}}(\xi ; w)}
$$

and its static limit

$$
\left\langle\mathcal{F}\left(s_{0}, \xi\right)\right\rangle=\frac{2 g\left(s_{0}, \xi\right)}{\chi_{\mathrm{eff}}(\xi ; 0)} .
$$

$\chi_{\text {eff }}(\xi ; w)$ depends on pressure, temperature, composition, wavelength, and in addition on the velocity gradient $w$. Since these are all local quantities, the effective extinction coefficient can be calculated once for all, i.e. independently from the specific application. However, while all thermodynamic quantities including the usual extinction coefficient $\chi(\xi)$ are isotropic in the "natural" comoving frame, in general $w(\boldsymbol{n})=\boldsymbol{n} \cdot \nabla(\beta \cdot \boldsymbol{n})(\mathrm{I}: 33)$ and hence $\chi_{\mathrm{eff}}(\xi ; w)$ are anisotropic. This anisotropy may result in a component of the flux vector which is perpendicular to the temperature gradient (cf. Paper II, Sect. 5). The anisotropy, however, enters the radiative quantities only via $w$ so that we restrict ourselves in this paper to considering the dependencies on $w$. The problem of the direction of the flux vector will be taken up in Paper IV of this series.

\subsection{Radiative flux for Poisson distributed lines}

We now apply the Poisson point process in order to obtain the expectation value of the monochromatic radiative flux (17) in the diffusion limit. The characteristic expression to be evaluated is the expectation value of $\exp (-\bar{X} \cdot s)$ as given by Eq. (10).
The expectation value of the radiative flux (17) then reads

$$
\left\langle\mathcal{F}\left(s_{0}, \xi ; w\right)\right\rangle=2 g\left(s_{0}, \xi\right) \int_{0}^{\infty} \mathrm{e}^{-\chi_{\mathrm{c}}(\xi) \cdot s} \Omega(\xi, \xi-w s ; w) \mathrm{d} s,
$$

and the corresponding static flux

$$
\left\langle\mathcal{F}\left(s_{0}, \xi\right)\right\rangle=2 g\left(s_{0}, \xi\right) \int_{0}^{\infty} \mathrm{e}^{-\chi_{\mathrm{c}}(\xi) s} \Omega(\xi, \xi ; 0) \mathrm{d} s
$$

with its line contribution given by Eq. (11). It should be kept in mind that $\Omega(\xi, \xi-w s ; w)$ and $\Omega(\xi, \xi ; 0)$ depend also on $s$.

\subsection{Radiative flux in terms of opacity distribution functions}

With the definition of the opacity distribution function as a probability, the relevant expectation value $\exp (-\bar{X} \cdot s)$ in the radiation transfer equation becomes

$$
\begin{aligned}
\Omega(\xi, \xi-w s ; w) & =\left\langle\mathrm{e}^{-\bar{X}(\xi ; w s) \cdot s}\right\rangle \\
& =\int_{0}^{\infty} \mathrm{e}^{-\bar{x} \cdot s} \tilde{p}(\bar{x} ; \xi, w s) \mathrm{d} \bar{x}
\end{aligned}
$$

and hence the expectation value of the monochromatic diffusion flux

$$
\begin{aligned}
& \left\langle\mathcal{F}\left(s_{0}, \xi ; w\right)\right\rangle=\frac{2 g\left(s_{0}, \xi\right)}{\chi_{\text {eff }}(\xi ; w)} \\
& \quad=2 g\left(s_{0}, \xi\right) \int_{0}^{\infty} \int_{0}^{\infty} \mathrm{e}^{-\left[\chi_{\mathrm{c}}(\xi)+\bar{x}\right] \cdot s} \tilde{p}(\bar{x} ; \xi, w s) \mathrm{d} \bar{x} \mathrm{~d} s .
\end{aligned}
$$

For the static case $\bar{x}$ has to be replaced by $x$ and $\tilde{p}$ by $p$. Furthermore, the integration over $s$ can here be carried out since $p$ does not depend on $s$, yielding for the reciprocal monochromatic effective extinction

$\frac{1}{\chi_{\mathrm{eff}}(\xi ; 0)}=\int_{0}^{\infty} \frac{p(x ; \xi)}{\chi_{\mathrm{c}}(\xi)+x} \mathrm{~d} x$

\subsection{Connection}

The central connection between the two concepts of the generalized opacity distribution functions and the presentation of lines by the Poisson point process, relevant for the expectation value of the monochromatic flux, is given the pair of equations (Baschek et al. 2001)

$$
\begin{aligned}
& \left\langle\mathrm{e}^{-\bar{X}(\xi ; \Delta) \cdot i k}\right\rangle=\int_{-\infty}^{\infty} \mathrm{e}^{-\bar{x} \cdot i k} \tilde{p}(\bar{x} ; \xi, \Delta) \mathrm{d} \bar{x} \\
& \tilde{p}(\bar{x} ; \xi, \Delta)=\frac{1}{2 \pi} \int_{-\infty}^{\infty} \mathrm{e}^{i k \bar{x}} \cdot\left\langle\mathrm{e}^{-\bar{X}(\xi ; \Delta) \cdot i k}\right\rangle \mathrm{d} k
\end{aligned}
$$

with $\Delta=w s$ and the characteristic function

$$
\begin{aligned}
& \left\langle\mathrm{e}^{-\bar{X}(\xi ; \Delta) \cdot i k}\right\rangle= \\
& \quad \exp \left(\int_{\Theta} \int_{-\infty}^{\infty} \varrho(\hat{\xi}, \vartheta)\left\{\mathrm{e}^{-\frac{i k}{\Delta} \int_{\xi-\Delta}^{\xi} \chi(\hat{\xi}, \vartheta, \zeta-\hat{\xi}) \mathrm{d} \zeta}-1\right\} \mathrm{d} \hat{\xi} \mathrm{d} \vartheta\right) .
\end{aligned}
$$

The characteristic function - related to $\Omega(\xi, \xi-w s ; w)$ (Eq. (10)) - is thus determined by $\varrho$ and $\chi$, and for a given $\Omega$ we can calculate the corresponding $\tilde{p}$. Conversely, $\tilde{p}$ determines the characteristic function of $\tilde{p}$, i.e. essentially $\Omega$, but $n o t$ the two basic functionals $\varrho$ and $\chi$ separately. 


\section{Radiative acceleration in the diffusion approximation}

According to Eq. (I:43) the expectation value of the monochromatic radiative acceleration is given by

$\left\langle a_{\mathrm{rad}}\left(s_{0}, \xi ; w\right)\right\rangle=\frac{1}{c}\left\langle\chi(\xi) \cdot \mathcal{F}\left(s_{0}, \xi ; w\right)\right\rangle$

with the extinction coefficient being $\chi(\xi)=\chi_{\mathrm{c}}(\xi)+X(\xi)$ (Eq. (1)). Since the integration over $s$ can be interchanged with the operator for the expectation value and since $X$ does not depend on $s$, we obtain

$$
\begin{aligned}
\left\langle a_{\mathrm{rad}}\left(s_{0}, \xi ; w\right)\right\rangle= & \frac{2 g\left(s_{0}, \xi\right)}{c} \int_{0}^{\infty} \mathrm{e}^{-\chi_{\mathrm{c}}(\xi) \cdot s} \\
& \times\left[\chi_{\mathrm{c}}(\xi)\left\langle\mathrm{e}^{-\bar{X}(\xi ; w s) \cdot s}\right\rangle+\left\langle X(\xi) \cdot \mathrm{e}^{-\bar{X}(\xi ; w s) \cdot s}\right\rangle\right] \mathrm{d} s .
\end{aligned}
$$

The expectation value of the flux $\langle\mathcal{F}\rangle$, or that of $\exp (-\bar{X} s)$, is known from Eq. (21) so that the problem is reduced to the evaluation of the expectation value of the product of the line extinction coefficient $X$ times the characteristic expression $\exp (-\bar{X} \cdot s)$ which, unfortunately, cannot be calculated from the knowledge of the opacity distribution function alone, i.e. without the use of a specific stochastic model.

On the other hand, in the static case $\bar{X}(\xi ; w s) \rightarrow X[\xi)$ so that the integration over $s$ can be carried out directly and independently of any assumption on the line distribution yielding

$$
\begin{aligned}
\left\langle a_{\mathrm{rad}}\left(s_{0}, \xi\right)\right\rangle & =\frac{2 g\left(s_{0}, \xi\right)}{c}\left\langle\int_{0}^{\infty}\left[\chi_{\mathrm{c}}(\xi)+X(\xi)\right] \cdot \mathrm{e}^{-\left[\chi_{\mathrm{c}}(\xi)+X(\xi)\right]} \mathrm{d} s\right\rangle \\
& =\frac{2 g\left(s_{0}, \xi\right)}{c}=\frac{2}{c} \frac{\partial B(T, \xi)}{\partial T} \frac{\partial T}{\partial s}
\end{aligned}
$$

(cf. I:22). Thus in the static diffusion limit $\left\langle a_{\text {rad }}\right\rangle$ does not directly depend on the extinction coefficient, but is solely determined by the temperature gradient which, however, in turn may indirectly be influenced by the extinction via the energy balance in the medium. We point out that this result for the expectation value $\left\langle a_{\mathrm{rad}}\right\rangle$ is identical to that for the deterministic radiative acceleration $a_{\text {rad }}$, which follows from $a_{\text {rad }} \propto \chi \mathcal{F}$ and $\mathcal{F} \propto 1 / \chi(\mathrm{cf} . \mathrm{I}: 17,18)$.

In contrast to the static case, we have to choose a particular stochastic distribution for the general, differentially moving case in order to evaluate the expectation value of the radiative acceleration. Assuming now the Poisson point process, we can employ the procedure described in Appendix A based on the identity (A.4) and calculate the expectation value of this product. With Eq. (A.5) we obtain

$$
\begin{aligned}
& \left\langle a_{\text {rad }}\left(s_{0}, \xi ; w\right)\right\rangle= \\
& \quad \frac{2 g\left(s_{0}, \xi\right)}{c} \int_{0}^{\infty} \mathrm{e}^{-\chi_{\mathrm{c}}(\xi) \cdot s} \Omega(\xi, \xi-w s ; w) \cdot\left[\chi_{\mathrm{c}}(\xi)+\tilde{\mathcal{E}}(\chi)\right] \mathrm{d} s .
\end{aligned}
$$

Although we have calculated the static case already directly (Eq. (31)), it is nevertheless useful to regain this result also for Poisson distributed lines as it provides some insight into the general case as well. In the static limit of Eq. (32),

$$
\begin{aligned}
& \left\langle a_{\text {rad }}\left(s_{0}, \xi\right)\right\rangle= \\
& \quad \frac{2 g\left(s_{0}, \xi\right)}{c} \int_{0}^{\infty} \mathrm{e}^{-\chi_{\mathrm{c}}(\xi) \cdot s} \Omega(\xi, \xi ; 0) \cdot\left[\chi_{\mathrm{c}}(\xi)+\mathcal{E}(\chi)\right] \mathrm{d} s,
\end{aligned}
$$

the integral over $s$ has the value 1 since the integrand is a total differential, i.e.

$$
\begin{aligned}
\mathrm{e}^{-\chi_{\mathrm{c}}(\xi) \cdot s} \cdot \Omega(\xi, \xi ; 0) \cdot\left[\chi_{\mathrm{c}}(\xi)\right. & +\mathcal{E}(\chi)]= \\
& -\frac{\partial}{\partial s}\left[\mathrm{e}^{-\chi_{\mathrm{c}}(\xi) \cdot s} \cdot \Omega(\xi, \xi ; 0)\right] .
\end{aligned}
$$

Note that $\partial \Omega(\xi, \xi ; 0) / \partial s=-\Omega(\xi, \xi ; 0) \cdot \mathcal{E}(\chi)$ according to Eqs. (11) and (14), and that $\Omega(\xi, \xi ; 0)=1$ for $s=0$.

Other than in the static case, a relation corresponding to (34) does not hold for the general moving case as $\partial \Omega(\xi, \xi-$ $w s ; w) / \partial s \neq-\Omega(\xi, \xi-w s ; w) \cdot \tilde{\mathcal{E}}(\chi)$ so that the integrand is not a total differential. The difference between the two terms, however, is involved: in the expression $\chi \cdot \exp (-\chi s)$ occurring in the integral over $\hat{\xi}$ of $\tilde{\mathcal{E}}(\chi)$ the extinction coefficient is $\chi=\chi(\hat{\xi}, \vartheta, \xi-\hat{\xi})$. On the other hand, the differentiation of $\Omega$ with respect to $s$ yields a very similar integral over $\hat{\xi}$, with the only difference that the first factor is to be taken at a wavelength shifted by $w s$, i.e. $\chi=\chi((\hat{\xi}, \vartheta, \xi-w s-\hat{\xi})$ whereas the argument of $\chi$ in the exponential is not shifted. Thus, if $\varrho(\xi, \vartheta)$ does not vary strongly with $\xi$, then approximately

$$
\begin{aligned}
\mathrm{e}^{-\chi_{\mathrm{c}}(\xi) \cdot s} \cdot \Omega(\xi, \xi-w s ; w) \cdot\left[\chi_{\mathrm{c}}(\xi)+\tilde{\mathcal{E}}(\chi)\right] \approx \\
-\frac{\partial}{\partial s}\left[\mathrm{e}^{-\chi_{\mathrm{c}}(\xi) \cdot s} \cdot \Omega(\xi, \xi-w s ; w)\right],
\end{aligned}
$$

so that the integral over $s$ in (32) becomes 1. Hence the radiative acceleration is approximately given by the static expression (31) which does not directly depend on the extinction coefficient. Expansion of (35) in terms of $w$ shows that - for slowly varying $\varrho$ - the difference from the static expression is at most of the order of $w^{4}$ (cf. also Sect. 6.3).

This result implies that in the diffusion approximation the radiative acceleration is hardly influenced by spectral lines even if the medium is differentially moving. Numerical experiments have shown that indeed $\left\langle a_{\text {rad }}\left(s_{0}, \xi ; w\right)\right\rangle=2 g\left(s_{0}, \xi\right) / c=$ $a_{\text {rad }}\left(s_{0}, \xi\right)$ is fulfilled to a very good approximation (i.e. relative error less than $10^{-4}$ ).

\section{Limit of large velocity gradients}

In Paper II we derived and discussed the flux and the radiative acceleration in the diffusion approximation for the limiting cases of small and large velocity gradients $|w|$ for a deterministic line extinction coefficient, and defined the validity of these expressions by (II:1, 3). In this and the following section we calculate the corresponding expressions for a stochastic line distribution according to the Poisson point process.

In order to derive the expectation value of the flux in the limit of large $|w|$ we first note that the argument of the innermost exponential function in Eq. (10) gets small since the integral over $\zeta$ has an upper bound due to the normalization of the line profile function. Thus for sufficiently large $|w|$ an expansion to first order provides a good approximation. If we furthermore assume that the line density $\varrho$ neither varies over a line nor over $w s$ (where $s$ is the geometrical distance corresponding to a continuum optical depth of unity) and, for simplicity, adopt 
for all lines the same intrinsic width $\gamma_{0}$ and consider only the distribution of the line strengths $A$, i.e. $\varrho(\hat{\xi}, \vartheta)=\varrho(\hat{\xi}, A)$, then

$$
\begin{aligned}
\Omega_{\infty} & \equiv \lim _{|w| \rightarrow \infty} \Omega(\xi, \xi-w s ; w) \\
& =\exp \left(-\frac{1}{w} \int_{\Theta} \int_{-\infty}^{\infty} \int_{\xi-w s}^{\xi} \varrho(\hat{\xi}, \vartheta) \chi(\hat{\xi}, \vartheta, \hat{\xi}-\zeta) \mathrm{d} \zeta \mathrm{d} \hat{\xi} \mathrm{d} \vartheta\right) \\
& =\exp \left(-\int_{A} A \varrho(\xi, A) \mathrm{d} A \cdot s\right)
\end{aligned}
$$

since, after interchanging the integrations over $\zeta$ and $\hat{\xi}$, these integrals can be evaluated yielding first $\int_{-\infty}^{\infty} \chi \mathrm{d} \hat{\xi}=A$ (Eq. (7)), and then $A \cdot w s$ for the subsequent integration over $\zeta$. Finally, according to Eq. (21),

$$
\begin{aligned}
\lim _{|w| \rightarrow \infty}\left\langle\mathcal{F}\left(s_{0}, \xi ; w\right)\right\rangle & =2 g\left(s_{0}, \xi\right) \int_{0}^{\infty} \mathrm{e}^{-\chi_{\mathrm{c}} s} \Omega_{\infty} \mathrm{d} s \\
& =\frac{2 g\left(s_{0}, \xi\right)}{\chi_{\mathrm{c}}(\xi)+\int_{A} A \varrho(\xi, A) \mathrm{d} A} .
\end{aligned}
$$

The effective monochromatic extinction coefficient $\chi_{\text {eff }}(\xi ; w)$, defined in Eq. (17), is thus - in the limit of large $|w|$ - the sum of the continuous extinction and the average of the line extinction over the line strengths around $\xi$.

The limit of the radiative acceleration (Eq. (32)) for large $|w|$ can be obtained by expansion in terms of $w$ analogously to that for the flux. Since

$$
\begin{aligned}
\Omega_{\infty} \cdot\left[\chi_{\mathrm{c}}(\xi)+\right. & \left.\lim _{|w| \rightarrow \infty} \tilde{\mathcal{E}}(\chi)\right]= \\
& \mathrm{e}^{-\int_{A} A \varrho(\xi, A) \mathrm{d} A \cdot s} \cdot\left[\chi_{\mathrm{c}}(\xi)+\int_{A} A \varrho(\xi, A) \mathrm{d} A\right],
\end{aligned}
$$

we find

$$
\begin{aligned}
& \lim _{|w| \rightarrow \infty}\langle\left.a_{\mathrm{rad}}\left(s_{0}, \xi ; w\right)\right\rangle=\frac{2 g\left(s_{0}, \xi\right)}{c} \int_{0}^{\infty} \mathrm{e}^{-\left[\chi_{\mathrm{c}}(\xi) \cdot s+\int_{A} A \varrho(\xi, A) \mathrm{d} A\right]} \\
& \times\left[\chi_{\mathrm{c}}(\xi)+\int_{A} A \varrho(\xi, A) \mathrm{d} A\right] \mathrm{d} s=\frac{2 g\left(s_{0}, \xi\right)}{c},
\end{aligned}
$$

i.e. the radiative acceleration for very large $|w|$ is equal to that in the static case (Eq. (31)).

\section{Limit of small velocity gradients}

First the flux or effective extinction, respectively, is expressed for small $w$ as expansion of the generalized opacity distribution. Then, in the following two subsections, the expectation values of the flux and the radiative acceleration are derived for Poisson distributed lines.

\subsection{Effective extinction}

If we assume that the generalized opacity distribution function can be approximated by

$$
\tilde{p}(\bar{x} ; \xi, \Delta) \approx \tilde{p}(\bar{x} ; \xi, 0)+\Delta \cdot \tilde{p}_{1}(\bar{x} ; \xi)+\frac{1}{2} \Delta^{2} \cdot \tilde{p}_{2}(\bar{x} ; \xi)
$$

with $\Delta=w s$, the integrations in Eq. (24) can be interchanged, and the integration over $s$ be performed. Furthermore, since in the construction of the generalized opacity distribution function one considers a region of many lines and since the opacities at the starting and end points are identical, a linear term cannot exist. We thus obtain

$\frac{1}{\chi_{\mathrm{eff}}(\xi ; w)}=\int_{0}^{\infty} \frac{\tilde{p}(\bar{x} ; \xi, 0)}{\chi_{\mathrm{c}}(\xi)+\bar{x}} \mathrm{~d} \bar{x}+\int_{0}^{\infty} \frac{\tilde{p}_{2}(\bar{x} ; \xi)}{\left[\chi_{\mathrm{c}}(\xi)+\bar{x}\right]^{3}} \mathrm{~d} \bar{x} \cdot w^{2}$.

After integration over wavelength we can identify the first term as the static Rosseland mean opacity and the additional term as correction that describes the consequences of the velocity field.

\subsection{Radiative flux}

There are two ways to derive the expression that shows the dependence of the expectation value of the monochromatic flux in the limit of small velocity gradients: in the first one we expand the integrand of Eq. (21) in terms of $w$, or - in the second way - we use Eq. (II:17) and calculate the expectation values of the terms involved. Since the first way is more straightforward we describe it subsequently in the main part of the paper whereas the second way is followed in Appendix B.

Using a Taylor expansion of the integrand of Eq. (21) to second order around $w=0$, we can write the expectation value of the flux

$$
\begin{aligned}
& \left\langle\mathcal{F}\left(s_{0}, \xi ; w\right)\right\rangle= \\
& \quad 2 g\left(s_{0}, \xi\right) \int_{0}^{\infty} \mathrm{e}^{-\chi_{\mathrm{c}}(\xi) s} \cdot \Omega_{0} \cdot\left[1+\omega_{1} \cdot w+\frac{1}{2} \omega_{2} \cdot w^{2}\right] \mathrm{d} s
\end{aligned}
$$

with $\Omega_{0} \equiv \Omega(\xi, \xi ; 0)$ (Eq. (11)),

$\omega_{1}=\left.\frac{1}{\Omega_{0}} \cdot \frac{\partial \Omega(\xi, \xi-w s ; w)}{\partial w}\right|_{(\xi, \xi ; 0)}=\frac{s^{2}}{2} \mathcal{E}\left(\chi^{\prime}\right)$,

and

$$
\begin{aligned}
\omega_{2} & =\left.\frac{1}{\Omega_{0}} \cdot \frac{\partial^{2} \Omega(\xi, \xi-w s ; w)}{\partial w^{2}}\right|_{(\xi, \xi ; 0)} \\
& =\frac{s^{4}}{4}\left[\left(\mathcal{E}\left(\chi^{\prime}\right)\right)^{2}+\mathcal{E}\left(\left(\chi^{\prime}\right)^{2}\right)\right]-\frac{s^{3}}{3} \mathcal{E}\left(\chi^{\prime \prime}\right)
\end{aligned}
$$

where the abbreviation $\mathcal{E}$ is defined by Eq. (14). Here $\chi^{\prime}$ and $\chi^{\prime \prime}$ denote the first and second derivative of $\chi(\hat{\xi}, \vartheta, \xi-\hat{\xi})$ with respect to $\xi$. We point out that $\Omega_{0}$ and $\mathcal{E}$ and hence $\omega_{1}$ and $\omega_{2}$ depend not only on $\xi$ but also on $s$ and cannot be taken out of the respective integrals in the expression for the monochromatic flux.

The coefficients can be considerably simplified if $\varrho(\hat{\xi}, \vartheta)$ can be taken out of the integrals over $\hat{\xi}$ in the expressions $\mathcal{E}$ (cf. Eq. (15)), an assumption which is reasonable as $\varrho$ has to be a slowly varying function (Sect. 2.2). Then

$\omega_{1}=0$,

$$
\begin{aligned}
\omega_{2} & =\frac{s^{4}}{4} \mathcal{E}\left(\left(\chi^{\prime}\right)^{2}\right)-\frac{s^{3}}{3} \mathcal{E}\left(\chi^{\prime \prime}\right) \\
& =\frac{s^{3}}{3}\left[\mathcal{E}\left(s\left(\chi^{\prime}\right)^{2}-\chi^{\prime \prime}\right)-\frac{s}{4} \mathcal{E}\left(\left(\chi^{\prime}\right)^{2}\right)\right] \\
& =-\frac{s^{4}}{12} \mathcal{E}\left(\left(\chi^{\prime}\right)^{2}\right)
\end{aligned}
$$


where the identities $s \cdot \chi^{\prime} \mathrm{e}^{-\chi \cdot s} \equiv-\left(\mathrm{e}^{-\chi \cdot s}\right)^{\prime}$ and $\left[s \cdot\left(\chi^{\prime}\right)^{2}-\right.$ $\left.\chi^{\prime \prime}\right] \mathrm{e}^{-\chi \cdot s} \equiv-\left(\chi^{\prime} \mathrm{e}^{-\chi \cdot s}\right)^{\prime}$, respectively, have been employed so that $\mathcal{E}\left(\chi^{\prime}\right)$ and $\mathcal{E}\left(s \cdot\left(\chi^{\prime}\right)^{2}-\chi^{\prime \prime}\right)$ vanish. For the evaluation note that the prime indicates differentiation with respect to $\xi$ but that $\mathcal{E}$ comprises an integration over $\hat{\xi}$. Since, however, $\mathrm{d} \hat{\xi}=-\mathrm{d} \xi$, the above identities are valid for differentiation with respect to $\hat{\xi}$ as well.

The result that $\omega_{1}=0$ could also have been inferred directly from the symmetry of the expectation value (Eq. (12)) with respect to the sign of $w$ which allows only contributions of even powers of $w$.

\subsection{Radiative acceleration}

In order to obtain an expression for the radiative acceleration in media with small velocity gradients we proceed in the same way as for the flux, i.e. we expand the integrand of Eq. (30) to second power in $w$ and find

$$
\begin{aligned}
& \left\langle a_{\text {rad }}\left(s_{0}, \xi ; w\right)\right\rangle= \\
& \quad \frac{2 g\left(s_{0}, \xi\right)}{c}\left(1+\int_{0}^{\infty} \mathrm{e}^{-\chi_{\mathrm{c}}(\xi) \cdot s}\left[\tilde{\omega}_{1} \cdot w+\frac{1}{2} \tilde{\omega}_{2} \cdot w^{2}\right] \mathrm{d} s\right)
\end{aligned}
$$

with the coefficients

$$
\tilde{\omega}_{1}=\frac{s^{2}}{2} \Omega_{0} \cdot\left\{\left[\chi_{\mathrm{c}}+\mathcal{E}(\chi)\right] \mathcal{E}\left(\chi^{\prime}\right)+\mathcal{E}\left(\chi \chi^{\prime}\right)\right\}
$$

and

$$
\begin{aligned}
\tilde{\omega}_{2}= & \frac{s^{4}}{4} \Omega_{0} \cdot\left\{\left[\chi_{\mathrm{c}}+\mathcal{E}(\chi)\right] \cdot\left[\left(\mathcal{E}\left(\chi^{\prime}\right)\right)^{2}+\mathcal{E}\left(\left(\chi^{\prime}\right)^{2}\right)\right]\right. \\
& \left.+\mathcal{E}\left(\chi\left(\chi^{\prime}\right)^{2}\right)+2 \mathcal{E}\left(\chi^{\prime}\right) \cdot \mathcal{E}\left(\chi \chi^{\prime}\right)\right\} \\
- & \frac{s^{3}}{3} \Omega_{0} \cdot\left\{\left[\chi_{\mathrm{c}}+\mathcal{E}(\chi)\right] \cdot \mathcal{E}\left(\chi^{\prime \prime}\right)+\mathcal{E}\left(\chi \chi^{\prime \prime}\right)\right\}
\end{aligned}
$$

where $\Omega_{0}=\Omega(\xi, \xi ; 0)$ as well as $\tilde{\omega}_{1}$ and $\tilde{\omega}_{2}$ depend also on the variable $s$.

If $\varrho$ can be taken out of the $\hat{\xi}$-integrals of the $\mathcal{E}$, these coefficients can be drastically simplified: we have already shown (Eqs. $(45,46))$ that $\mathcal{E}\left(\chi^{\prime}\right)=0$ and $s \cdot \mathcal{E}\left(\left(\chi^{\prime}\right)^{2}\right)-\mathcal{E}\left(\chi^{\prime \prime}\right)=0$. Since furthermore the identity $\left[\chi^{\prime}-s \cdot \chi \chi^{\prime}\right] \mathrm{e}^{-\chi \cdot s}=\left(\chi \mathrm{e}^{-\chi \cdot s}\right)^{\prime}$ holds, $\mathcal{E}\left(\chi \chi^{\prime}\right)=0$ and hence $\tilde{\omega}_{1}=0$. Again, as for the coefficient $\omega_{1}$ for the flux, this result could also be obtained directly from the symmetry of the expression (12). By this argument, of course, also the coefficient of the $w^{3}$-term of the radiative acceleration vanishes. Finally, by employing identities of the type

$$
\begin{aligned}
& \frac{\partial}{\partial s}\left[\mathrm{e}^{-\chi_{\mathrm{c}} \cdot s} \Omega_{0} s^{k} \mathcal{E}(Z)\right]= \\
& \quad-\mathrm{e}^{-\chi_{\mathrm{c}} \cdot s} \Omega_{0} s^{k}\left\{\left[\chi_{\mathrm{c}}+\mathcal{E}(\chi)-k / s\right] \cdot \mathcal{E}(Z)+\mathcal{E}(\chi Z)\right\}
\end{aligned}
$$

with $k=\{3,4\}$ and $Z=\left\{\chi^{\prime \prime},\left(\chi^{\prime}\right)^{2}\right\}$, it can be shown that the integral $\int_{0}^{\infty} \exp \left(-\chi_{\mathrm{c}} s\right) \tilde{\omega}_{2} \mathrm{~d} s$ in Eq. (47) and hence the $w^{2}$-term vanishes.

Thus for Poisson distributed lines the radiative acceleration for small $w$ deviates from the static value by at most a term of the order of $w^{4}$ in agreement with the result obtained by expansion of the expression (35) in terms of $w$ (cf. Sect. 4).

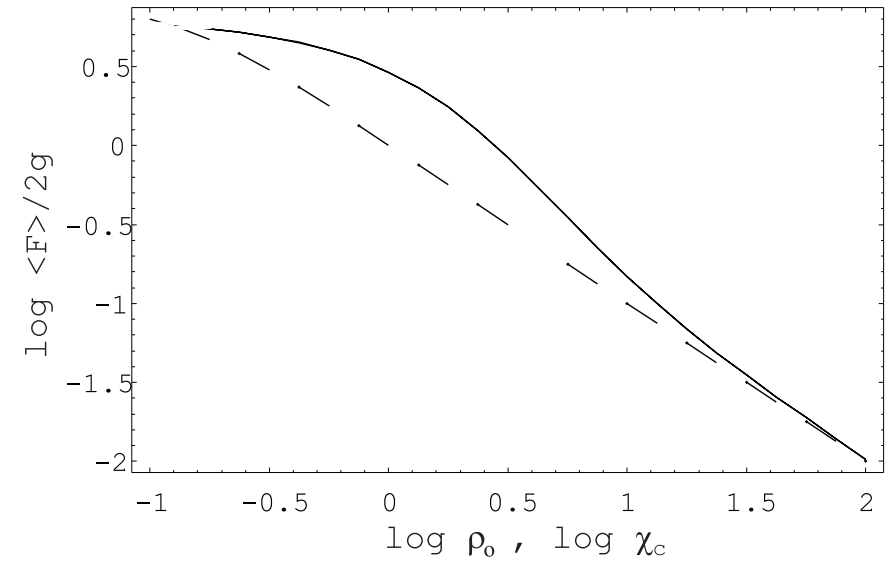

Fig. 1. Comparison of the consequences of an increase in the line absorption (by means of an increase in the line density $\varrho$, full curve, and of continuous extinction $\chi_{\mathrm{c}}$, broken curve) for the expectation value of the monochromatic diffusion flux (in units of $2 g$ ), i.e. of $\left\langle\mathcal{F}\left(s_{0}, \xi ; w\right)\right\rangle /\left(2 g\left(s_{0}, \xi\right)\right)$. The broken curve is normalized to have the same asymptote as the full curve for large extinction. For the line extinction the additional parameters $\chi_{\mathrm{c}}=0.1, w=0.01, \gamma_{0}=0.1$, and $A_{0}=1$ are assumed.

\section{Examples for the monochromatic flux}

In this section we give some examples that illustrate the different consequences of continuous and line extinction for the flux and the variations with the line parameters. In order to facilitate the discussion we consider here only lines with identical shapes (to be more precise: lines of Lorentzian shapes with the same strength $A_{0}$ and width $\gamma_{0}$ ), and furthermore assume the line density to be wavelength-independent so that

$\varrho(\hat{\xi}, \vartheta)=\varrho_{0} \cdot \delta\left(A-A_{0}\right) \cdot \delta\left(\gamma-\gamma_{0}\right)$.

We stress that this simplification of the line density is solely assumed for the purpose of demonstrating the basic effects; for any practical application a suitable distribution of the line strengths should be adopted (whereas the intrinsic line width or damping constant may still be chosen to be the same for all lines). In view of the result of Learner (1982), the strength distribution may be approximated in a range $A \in\left[A_{1}, A_{2}\right]$ by a power law $f(A) \mathrm{d} A \propto A^{-\alpha} \mathrm{d} A$.

In Figs. 1 to 5 the vertical axes show the expectation value of the monochromatic radiative flux $\langle\mathcal{F}\rangle$ for $2 g=1$ or of the effective mean free path $1 / \chi_{\text {eff }}(\xi ; w)$ (cf. Eq. (17)).

In Fig. 1 the variation of $\langle\mathcal{F}\rangle$ is shown on a logarithmic scale as a function of mean line density and of the continuous extinction coefficient. The latter is well known from Rosseland's classical formula (cf. also Paper I).

Even for the non-extreme set of parameters used here the variations of the continuous and line extinctions lead to quite different results: when the line density is small, $\langle\mathcal{F}\rangle$ is only slightly affected (in particular when the continuous extinction dominates it does not influence the flux as is intuitively evident). However, when a critical line density is passed, there is a very strong dependence on $\varrho_{0}$ before the curves join due to saturation.

In Fig. 2 we have plotted the flux versus the line density with the constraint that the product of line density and strength, 


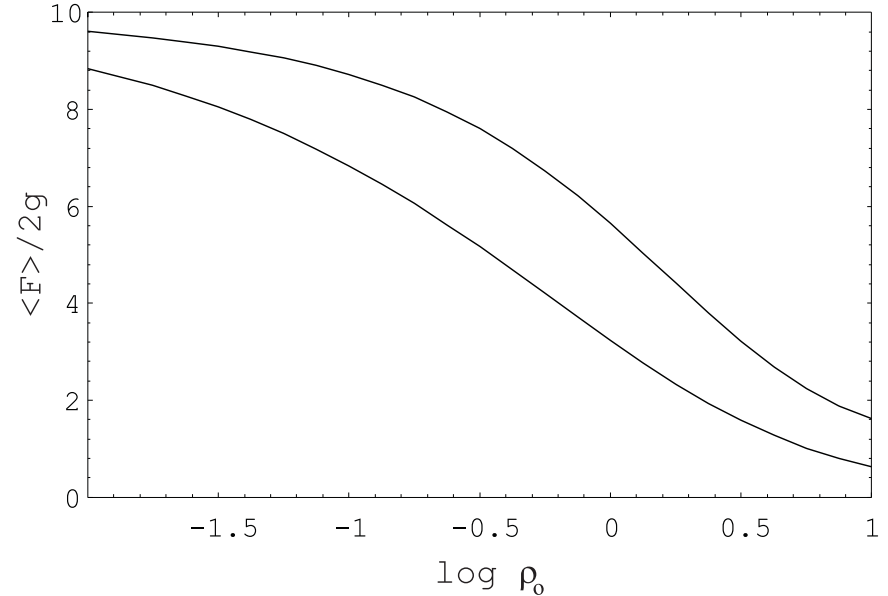

Fig. 2. Variation of $\left\langle\mathcal{F}\left(s_{0}, \xi ; w\right)\right\rangle /\left(2 g\left(s_{0}, \xi\right)\right)$ with the line density $\varrho_{0}$. The line strengths are assumed to be $A_{0}=1 / \varrho_{0}$ (upper curve) and $A_{0}=10 / \varrho_{0}$ (lower curve), i.e. along each curve the total absorption is kept constant. The graph indicates that many weak lines are more effective than a few strong ones producing the same total absorption. The additional parameters are $\chi_{\mathrm{c}}=0.1, w=0.05$, and $\gamma_{0}=0.01$.

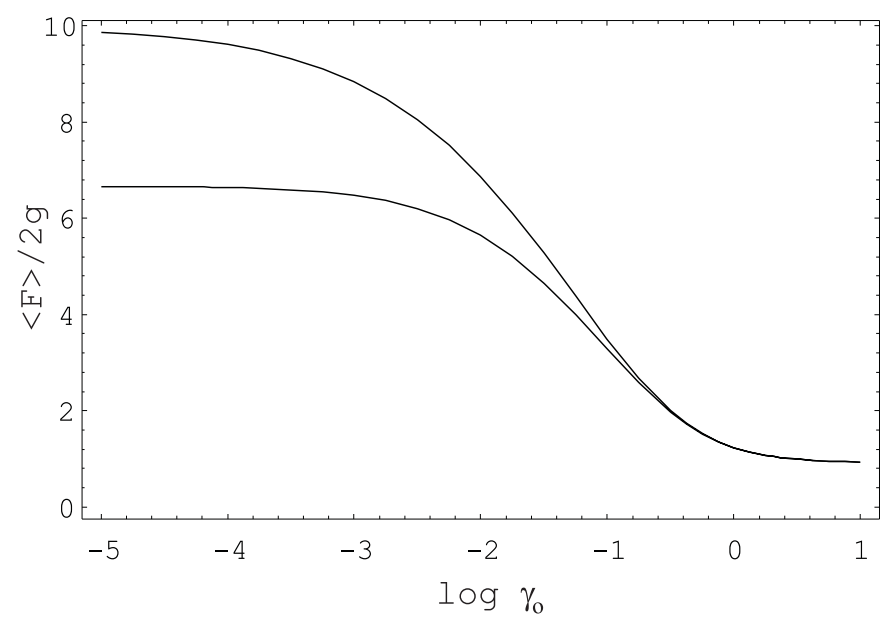

Fig. 3. Variation of $\left\langle\mathcal{F}\left(s_{0}, \xi ; w\right)\right\rangle /\left(2 g\left(s_{0}, \xi\right)\right)$ with the line width parameter $\gamma_{0}$ for velocity gradients $w=0.05$ (lower curve) and $w=$ 0.0005 (upper curve). The additional parameters are $\chi_{\mathrm{c}}=0.1, A_{0}=1$, and $\varrho_{0}=1$.

$\varrho_{0} A_{0}$, is constant. This includes as limiting cases on the one hand a few strong lines (small $\varrho_{0}$, large $A_{0}$ ), and on the other many weak lines (large $\varrho_{0}$, small $A_{0}$ ) having the same total absorption. It is seen that over a wide range few strong lines are less effective than many weak lines with the same $\varrho_{0} A_{0}$.

In Fig. 3 the influence of the damping is investigated for two velocity gradients: for very small and very high damping the flux depends very little on the precise value of $\gamma_{0}$ but for intermediate ranges (which is more extended for small gradients) $\gamma_{0}$ is of high importance. Note in particular that for sufficiently large damping the value of $\gamma_{0}$ is more important than that of $w$.

The consequences of the line strength parameter $A_{0}$ on the flux (Fig. 4) is essentially as expected in that stronger lines lead to a stronger reduction in the flux. But it should be noted that there is no general scaling when the velocity gradient is

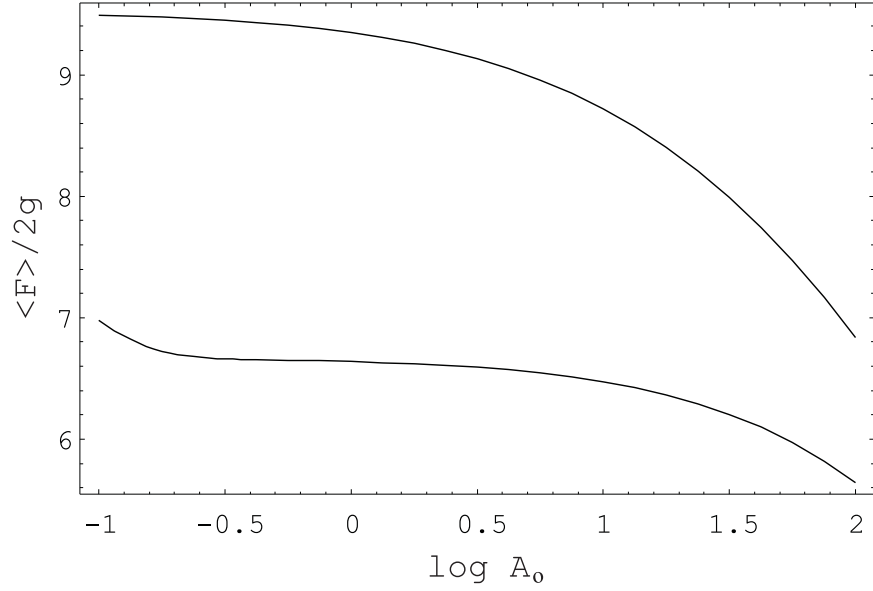

Fig. 4. Variation of $\left\langle\mathcal{F}\left(s_{0}, \xi ; w\right)\right\rangle /\left(2 g\left(s_{0}, \xi\right)\right)$ with the line strength $A_{0}$ for velocity gradients $w=0.05$ (lower curve) and $w=0.005$ (upper curve). The additional parameters are $\chi_{\mathrm{c}}=0.1, \gamma_{0}=0.0001$, and $\varrho_{0}=1$.

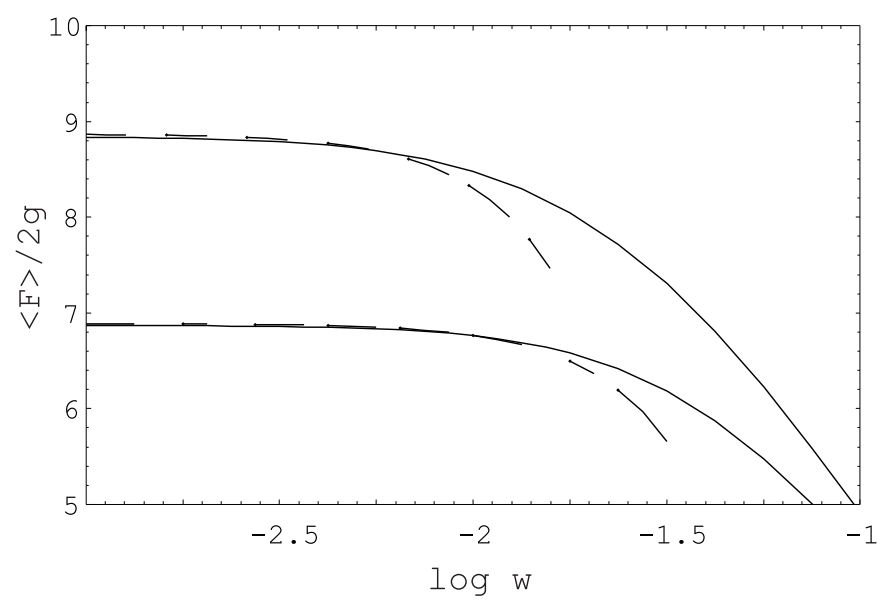

Fig. 5. Variation of $\left\langle\mathcal{F}\left(s_{0}, \xi ; w\right)\right\rangle /\left(2 g\left(s_{0}, \xi\right)\right)$ with the velocity gradient $w$ for line width parameters $\gamma_{0}=0.01$ (lower curve) and $\gamma_{0}=0.001$ (upper curve). For comparison the limit (42) for small $w$ is shown by thebroken curves. The additional parameters are $\chi_{\mathrm{c}}=0.1, A_{0}=1$, and $\varrho_{0}=1$.

changed, i.e. the effects of velocity and line strength do not factor in general as they do for small $w$ (cf. Sect 6.2).

The variation with the velocity gradient (Fig. 5) follows for small values the asymptotic formulae (42) well, but it is also evident that the approximations for small $w$ strongly underestimate the effects for larger gradients.

\section{Total radiative flux}

Integration of Eq. (17) over wavelength provides the expectation value of the total flux in the diffusion limit,

$$
\begin{aligned}
\left\langle\mathcal{F}_{\text {tot }}\left(s_{0} ; w\right)\right\rangle & =\int_{-\infty}^{\infty}\left\langle\mathcal{F}\left(s_{0}, \xi ; w\right)\right\rangle \mathrm{e}^{\xi} \mathrm{d} \xi \\
& =\int_{-\infty}^{\infty} \frac{2 g\left(s_{0}, \xi\right)}{\chi_{\mathrm{eff}}(\xi ; w)} \mathrm{e}^{\xi} \mathrm{d} \xi
\end{aligned}
$$


(I:45), and the generalization of the (reciprocal) Rosseland mean opacity of static to differentially moving media,

$\left\langle\frac{1}{\bar{\chi}_{\beta}\left(s_{0} ; w\right)}\right\rangle=\int_{-\infty}^{\infty} \frac{G\left(s_{0}, \xi\right)}{\chi_{\text {eff }}(\xi ; w)} \mathrm{d} \xi$

(I:49) with $G\left(s_{0}, \xi\right)$ being the weighting function defined in $(\mathrm{I}: 8)$.

The expectation value of the reciprocal conventional (static) Rosseland mean, $\bar{\chi}_{\mathrm{R}}\left(s_{0}\right) \equiv \bar{\chi}_{\beta}\left(s_{0} ; 0\right)$, is

$\left\langle\frac{1}{\bar{\chi}_{\mathrm{R}}\left(s_{0}\right)}\right\rangle=\int_{-\infty}^{\infty} \frac{G\left(s_{0}, \xi\right)}{\chi_{\text {eff }}(\xi ; 0)} \mathrm{d} \xi$.

In the limit of small velocity gradients the expectation value of the total flux in the diffusion limit can be expressed analogously to (II:18) as

$\left\langle\mathcal{F}_{\text {tot }}\left(s_{0} ; w\right)\right\rangle=\left\langle\mathcal{F}_{\text {tot }}\left(s_{0}\right)\right\rangle \cdot\left[1+\eta_{1}^{*}\left(s_{0}\right) \cdot w+\eta_{2}^{*}\left(s_{0}\right) \cdot w^{2}\right]$

where $\left\langle\mathcal{F}_{\text {tot }}\left(s_{0}\right)\right\rangle$ is the expectation value of the total static flux, and the coefficients $\eta_{1}, \eta_{2}$ are given by

$\eta_{m}^{*}\left(s_{0}\right)=\frac{1}{m} \cdot \frac{\int_{-\infty}^{\infty} g\left(s_{0}, \xi\right) \int_{0}^{\infty} \omega_{m}(\xi, s) \Psi(\xi, s) \mathrm{d} s \mathrm{~d} \xi}{\int_{-\infty}^{\infty} g\left(s_{0}, \xi\right) \int_{0}^{\infty} \Psi(\xi, s) \mathrm{d} s \mathrm{~d} \xi}$

$(m=1,2)$ with $\Psi(\xi, s)=\exp \left(-\chi_{\mathrm{c}}(\xi) s\right) \Omega(\xi, \xi ; 0) \mathrm{e}^{\xi}$. The coefficients $\eta_{m}$ are mean values of the $\omega_{m}$ over $s$ and $\xi$, weighted by the "static" function $\Psi(\xi, s)$.

For Poisson distributed lines it was shown in Sect. 6.2 that $\omega_{1}=0$ and $\omega_{2}<0$ and hence $\eta_{1}^{*}=0$ and $\eta_{2}^{*}<0$.

\section{Sign of the flux change due to motions}

It is of prime importance to find in which way the radiative flux is changed when a velocity field is added to a given static medium. Therefore we aim at the determination the sign of the difference

$\delta\langle\mathcal{F}\rangle=\left\langle\mathcal{F}\left(s_{0}, \xi ; w\right)\right\rangle-\left\langle\mathcal{F}\left(s_{0}, \xi\right)\right\rangle$.

Since we cannot find a general method to derive this sign for the presentation of the flux in terms of opacity distribution functions, we consider the expressions for Poisson distributed lines, i.e.

$\delta\langle\mathcal{F}\rangle=2 g\left(s_{0}, \xi\right) \int_{0}^{\infty} \mathrm{e}^{-\chi_{\mathrm{c}} s}[\Omega(\xi, \xi-w s ; w)-\Omega(\xi, \xi ; 0)] \mathrm{d} s$

with

$$
\begin{aligned}
\Omega(\xi, \xi-w s ; w)- & \Omega(\xi, \xi ; 0)= \\
{[\exp } & \left\{\int_{A} \varrho(\xi, A) \int_{-\infty}^{\infty}\left(\mathrm{e}^{-A U \cdot s}-1\right) \mathrm{d} \hat{\xi} \mathrm{d} A\right\} \\
& \left.-\exp \left\{\int_{A} \varrho(\xi, A) \int_{-\infty}^{\infty}\left(\mathrm{e}^{-A \Phi \cdot s}-1\right) \mathrm{d} \hat{\xi} \mathrm{d} A\right\}\right] .
\end{aligned}
$$

Without loss of generality we may choose the fluxes themselves, $g\left(s_{0}, \xi\right), s$, and $w$ to be positive. In addition we assume - as in Sect. 5 - that all lines have the same intrinsic width $\gamma_{0}$ and that $\varrho(\hat{\xi}, \vartheta)=\varrho(\hat{\xi}, A)$ can be taken out of the integral over $\hat{\xi}$. Here $\Phi=\Phi\left(\hat{\xi}, \gamma_{0}, \xi-\hat{\xi}\right)$ is the line profile (cf. Eq. (7)), and $U=U\left(\hat{\xi}, \xi, \Delta, \gamma_{0}\right)=\int_{\xi-\Delta}^{\xi} \Phi\left(\hat{\xi}, \gamma_{0}, \zeta-\hat{\xi}\right) \mathrm{d} \zeta / \Delta$ is the "average" over the interval $\Delta=w s$ of the single line profile which is characteristic for the Poisson point process (cf. Eq. (40) in Baschek et al. 2001). By their physical meaning $\chi_{\mathrm{c}}, A, \varrho, \Phi$, and $U$ are positive.

It seems to us very difficult to provide a general derivation of the sign of $\delta\langle\mathcal{F}\rangle$. However, in the limiting cases of large and small velocity gradients $w$ it is possible to determine the sign in a straightforward way.

For large velocity gradients, i.e. in the limit $|w| \rightarrow \infty$, the expectation value of the flux has already been evaluated in Sect. 5 upon the assumption that $\varrho(\hat{\xi}, A)$ can be taken out of the $\hat{\xi}$-integral. In this limit, $\int_{-\infty}^{\infty}\{\exp (-A U s)-1\} \mathrm{d} \hat{\xi} \rightarrow-A s$. Incidentally, the equivalent case of infinitely sharp lines and a finite interval $w s$ has been derived by Wehrse et al. (1998, their Eqs. (20) and (33)).

Now we consider the ratio $r$ of the $\hat{\xi}$-integrals for static case to that of the limit $|w| \rightarrow \infty$,

$$
\begin{aligned}
r & =\int_{-\infty}^{\infty}\left(\mathrm{e}^{-A \Phi\left(\hat{\xi}, \gamma_{0}, \xi-\hat{\xi}\right) \cdot s}-1\right) \mathrm{d} \hat{\xi} /(-A s) \\
& =\int_{-\infty}^{\infty} \frac{\mathrm{e}^{-A \Phi \cdot s}-1}{-A \Phi \cdot s} \cdot \Phi \mathrm{d} \hat{\xi}
\end{aligned}
$$

As $A, \Phi$, and $s$ are non-negative, the inequality $0<\left(\mathrm{e}^{-A \Phi s}-1\right) /(-A \Phi s) \leq 1$ holds for any $\hat{\xi}$ and $\xi$ so that, utilizing the normalization of the profile function, we obtain for any $A$ and $\xi$

$$
r \leq \int_{-\infty}^{\infty} \Phi \mathrm{d} \hat{\xi}=1 \quad \text { or } \quad \delta\langle\mathcal{F}\rangle \leq 0 .
$$

Note that both the numerator and denominator of (59) are negative.

For small velocity gradients $w$, according to Eq. (42) with $\omega_{1}=0$ (Eq. (45)), the change of the flux is given by

$\delta\langle\mathcal{F}\rangle=2 g\left(s_{0}, \xi\right) \int_{0}^{\infty} \mathrm{e}^{-\chi_{\mathrm{c}} s} \Omega_{0} \cdot \frac{1}{2} \omega_{2} \cdot w^{2} \mathrm{~d} s$.

Since $\Omega_{0}>0$ (cf. Eq. (11)), the sign of $\delta\langle\mathcal{F}\rangle$ is determined by that of $\omega_{2}$, and since $s^{4} \tilde{\mathcal{E}}\left(\left(\chi^{\prime}\right)^{2}\right) \geq 0$ (cf. Eq. (46)),

$\omega_{2} \leq 0 \quad$ and hence $\delta\langle\mathcal{F}\rangle \leq 0$.

Thus in the limit of small velocity gradients the expectation value of the flux depends on $w$ only in second order and is reduced compared to the static value as has been suggested already by the results for deterministic lines of Paper II.

Furthermore, we note that derivative of the flux change with respect to $w$ is given by

$$
\begin{aligned}
\frac{\partial}{\partial w} \delta\langle\mathcal{F}\rangle & =2 g\left(s_{0}, \xi\right) \int_{0}^{\infty} \mathrm{e}^{-\chi_{\mathrm{c}} s} \Omega_{0} \omega_{2} \mathrm{~d} s \cdot w \\
& <0 \quad \text { for } w>0
\end{aligned}
$$

and $>0$ for $w<0$. This again shows that for small $|w|$ the static flux is larger than the flux in a differentially moving medium. 
For the range of intermediate velocity gradients we have neither derived the sign of the flux change nor its derivative $\partial \delta\langle\mathcal{F}\rangle / \partial w$. However, our numerical evaluations assuming symmetric (Lorentzian) line profiles, $\varrho=\varrho_{0}$, and $A=A_{0}$ (see e.g. Fig. 5) strongly suggest that $\delta\langle\mathcal{F}\rangle\langle 0$ also holds in this intermediate range.

\section{Discussion and outlook}

The generalized opacity distribution function and the Poisson point process, two concepts of describing statistically a large number of spectral lines in the deep layers of differentially moving media, have been introduced in this paper. We have derived comoving-frame expressions for the expectation values of the monochromatic radiative flux and acceleration These expressions can in particular be used in radiation hydrodynamics. The presentation of the lines by a Poisson process in addition leads to general conclusions about the effects of the motions on the radiative quantities for essentially all kinds of reasonable spectral line profiles.

It should be noted that in actual modelling the formalism of Poisson distributed lines can be applied in a very flexible way. For example, it is not required that the statistical description comprises all spectral lines; if appropriate, a few stronger lines can be treated individually in addition, since the line extinction coefficient (1) is additive with respect to individual lines or line groups.

The discussion can be restricted to the monochromatic flux and radiative acceleration since no additional insight is gained from the corresponding wavelength-integrated expressions, and in practice, it is easy to obtain the total flux or the total radiative acceleration by simple numerical integrations over $\xi$ employing only few grid points.

The assumption of Poisson distributed lines implies that only the absolute value $|w|$ of the velocity gradient occurs in the radiative quantities and hence all effects due to the motions have to be even functions of $w$.

The central result of this paper, based on this assumption, is that the expectation value of the flux in a differentially moving medium is always reduced compared to the corresponding static value, i.e. $\delta\langle\mathcal{F}\rangle\langle 0$, or, equivalently, that the effective extinction is enhanced, i.e. that not only an expansion but also a contraction or collapse result in an enhanced effective extinction.

In the limit of small velocity gradients the effect of the differential motions depends on $w$ in second order. Although usually $w^{2}$ is small it is, however, multiplied by a factor which for narrow lines can become very large (cf. also the deterministic case in Paper II). The change in the effective opacity (cf. also Fig. 5) can reach a factor of about 2 which is much more than the present-day uncertainties of atomic and molecular data. In detail, the monochromatic flux depends - in addition to $w-$ on the continuous extinction $\chi_{\mathrm{c}}$, the line extinction $\chi$, and the line density $\varrho$, where the line contribution may be specified by the line strength $A$ and the line profile $\Phi=\Phi\left(\hat{\xi}, \gamma_{0}, \xi-\hat{\xi}\right)$ with its intrinsic width $\gamma_{0}$. We demonstrate the intricate behavior of the flux on these parameters for the simplest case, the limit of small velocity gradients $w$. In this case (Eq. (46)), the ratio $q$ of the term due to the differential motion to the static term at the wavelength $\xi$ is given by

$q(\xi)=\frac{w^{2}}{2} \cdot \frac{\int_{0}^{\infty} \omega_{2}(\xi, s) \cdot \mathrm{e}^{-\chi_{\mathrm{c}}(\xi) \cdot s} \Omega_{0}(\xi, s) \mathrm{d} s}{\int_{0}^{\infty} \mathrm{e}^{-\chi_{\mathrm{c}}(\xi) \cdot s} \Omega_{0}(\xi, s) \mathrm{d} s}$.

We recognize that $w^{2} / 2$ is multiplied by a particular average over $s$ of

$\omega_{2}(\xi, s)=-\frac{s^{4}}{12} \int_{A} A^{2} \varrho(\xi, A) \int_{-\infty}^{\infty}(\mathrm{d} \Phi / \mathrm{d} \hat{\xi})^{2} \mathrm{e}^{-A \Phi \cdot s} \mathrm{~d} \hat{\xi} \mathrm{d} A$,

weighted by $\exp \left(-\chi_{\mathrm{c}} s\right) \Omega_{0}$ which is a functional of $\chi_{\mathrm{c}}, \chi$ (or $A$ and $\Phi)$, and $\varrho$ (Eqs. $(11,58)$ ). The dominant contributions to the integrals over $s$ arise from the range about $s \simeq 1$. The coefficient $\omega_{2}$ is directly proportional to the line density $\varrho$ and to the integral essentially of the square of the $\hat{\xi}$-derivative of the line extinction coefficient (or of $A \cdot \Phi$ ) over all line positions $\hat{\xi}$.

In contrast to the flux, the radiative acceleration is hardly at all influenced by the Poisson distributed lines, for small velocity gradients the effect is at most of the order of $w^{4}$. In realistic situations, however, the energy balance or the conservation of the total flux $\mathcal{F}_{\text {total }}\left(s_{0}, w\right)$ is to be obeyed which leads to an increase of the temperature gradient and hence - according to Eq. (31) - indirectly to an increase of $a_{\mathrm{rad}}$.

The numerical evaluation of the integrals over $\hat{\xi}$ and $s$, required for the evaluation of the expectation values of the flux and the radiative acceleration, is quite intricate and has to be performed very carefully, since we experienced that e.g. standard routines of Mathematica and Maple with default parameters often gave unsatisfactory results.

So far we have considered only the case that the source function is given by the Planck function, i.e. in particular neglected line scattering. But we point out that it is possible to generalize our results so that scattering is included, mainly because the generalized opacity distribution function $\tilde{p}$ is independent of the mode of extinction (absorption or scattering) and can be derived for the Poisson point process by means of Levy's theorem. For example, for static media and coherent scattering we can write the expectation value of the flux as $\langle\mathcal{F}\rangle=\int_{0}^{\infty} \tilde{p}(\chi) \mathcal{F}(\chi) \mathrm{d} \chi$ and determine $\mathcal{F}(\chi)$ by numerical solution of the appropriate transfer equation for deterministic $\chi$.

In this paper we have concentrated on the stochastic representation of the spectral lines and obtained expressions for the flux or the effective extinction in a particular direction $\boldsymbol{n}$, essentially upon the assumption that the lines have a finite intrinsic width and that they obey a Poisson process. In the last Paper IV of this series we will briefly discuss the direction and the absolute value of the flux vector in 3D media, and furthermore apply our formalism also to the limiting case of infinitely sharp lines (Dirac $\delta$-profiles), a useful approximation which allows us to derive some further illustrative analytical results. We then will have available all relevant results in order to discuss the connection of our formalism, expressed in terms of the effective opacity $\chi_{\text {eff }}(\xi ; w)$ in the comoving frame to the observers frame concept of expansion opacity by Karp et al. (1977) which has frequently been used (incorrectly) in modelling of stars in the comoving frame (cf. Blinnikov 1996; Pinto \& Eastman 2000). 
In conclusion, we point out that our results can be applied to a manifold of astronomical objects, e.g. to the modelling of the deeper layers of novae, supernovae, Wolf-Rayet stars, miras, and accretion disks. They may also be useful to study the selective separation of elements due to radiative acceleration in the peculiar A-type stars or to the problem of the origin of stellar winds.

Acknowledgements. We are indebted to A. Mikelic and G. Shaviv for many helpful discussions. This work has been supported in part by the DFG (Sonderforschungsbereich 359/C2).

\section{Appendix A: Calculation of expectation values for Poisson distributed lines}

For lines which are stochastically distributed according to the Poisson point process, Wehrse et al. (1998) derived the characteristic expectation value $<\exp (-\bar{X}(\xi ; w s) \cdot s)>$ entering the expressions of the specific intensity and hence of the flux (Eq. (10)).

Since in this paper we want to calculate expectation values - in addition to that of the flux - also of the radiative acceleration (Sect. 4) as well as of expansions of these radiative quantities in terms of $w$ (Sect. 6, Appendix B), we consider instead of $\langle\exp (-\bar{X}(\xi ; w s) \cdot s)>$ the more general expression $<\exp (H(\xi ; w, s))>$ with

$H(\xi ; w, s)=\lambda \cdot X^{(i)}(\xi)+\mu \cdot X^{(j)}(\xi)-\bar{X}(\xi ; w s) \cdot s$.

Here $\lambda$ and $\mu$ are scalar parameters, and $X^{(i)}=\partial^{i} X / \partial \xi^{i}$ denotes the i-th partial derivative of $X(\xi)$ with respect to $\xi$. As long as $H(\xi ; w, s)$ is linear in $X$ and its derivatives, the result for $<\exp (-\bar{X} \cdot s)>$ of Wehrse et al. (1998) for Poisson distributed lines also holds for $\langle\exp (H)>$, i.e.

$\left\langle\mathrm{e}^{H(\xi ; w, s)}\right\rangle=\exp \left(\int_{\Theta} \int_{-\infty}^{\infty} \varrho(\hat{\xi}, \vartheta)\left\{\mathrm{e}^{h}-1\right\} \mathrm{d} \hat{\xi} \mathrm{d} \vartheta\right)$

with

$$
\begin{aligned}
h & \equiv h(\hat{\xi}, \vartheta, \xi-\hat{\xi} ; w, s) \\
& =\lambda \cdot \chi^{(i)}+\mu \cdot \chi^{(j)}-\frac{1}{w} \int_{\xi-w s}^{\xi} \chi(\hat{\xi}, \vartheta, \zeta-\hat{\xi}) \mathrm{d} \zeta
\end{aligned}
$$

$\left(\chi^{(i)}=\partial^{i} \chi / \partial \xi^{i}\right)$. This generalization allows the evaluation of all expectation values occurring in this paper by utilizing the identity

$$
\left.\frac{\partial^{m}}{\partial \lambda^{m}} \frac{\partial^{n}}{\partial \mu^{n}}\left\langle\mathrm{e}^{\lambda X^{(i)}+\mu X^{(j)}} V\right\rangle\right|_{\substack{\lambda=0 \\ \mu=0}}=\left\langle\left[X^{(i)}\right]^{m}\left[X^{(j)}\right]^{n} V\right\rangle
$$

which holds for an arbitrary function $V$; for the above generalization, $V=\exp (-\bar{X}(\xi, w s) s)$. Not that the differentiations of (A.2) with respect to $\lambda$ and/or $\mu$ affect only $\exp (h)$.

Of particular importance is the expectation value of $X \cdot \exp (-\bar{X} s)$, which is required for the calculation of the radiative acceleration (30). Setting $m=1$ and $n=0$ in Eq. (A.4), we obtain

$$
\begin{aligned}
\left\langle X \mathrm{e}^{-\bar{X} \cdot s}\right\rangle & =\left.\frac{\partial}{\partial \lambda}\left\langle\mathrm{e}^{\lambda X} \mathrm{e}^{-\bar{X}(\xi, w s) \cdot s}\right\rangle\right|_{\lambda=0} \\
& =\left\langle\mathrm{e}^{-\bar{X} \cdot s}\right\rangle \cdot \tilde{\mathcal{E}}(\chi)=\Omega(\xi, \xi-w s ; w) \cdot \tilde{\mathcal{E}}(\chi)
\end{aligned}
$$

where $\Omega$ and $\tilde{\mathcal{E}}(\chi)$ are defined by Eqs. (10) and (13), respectively.

\section{Appendix B: Alternative derivation of the radiative coefficients for small $|w|$}

Besides the "direct" way (Sects. 6.2, 6.3) we present here an alternative, in principle more general method of deriving the coefficients for the monochromatic flux and radiative acceleration for Poisson distributed lines.

According to the expansion in terms of $w$ (Eqs. (II:15) and (II:17)) the expectation value of the monochromatic radiative $f u x$ (17) for small velocity gradients is

$$
\begin{aligned}
\left\langle\mathcal{F}\left(s_{0}, \xi ; w\right)\right\rangle= & 2 g\left(s_{0}, \xi\right)\left[\left\langle\frac{1}{\chi_{\mathrm{c}}+X}\right\rangle-\left\langle\frac{1}{\chi_{\mathrm{c}}+X} \frac{\partial}{\partial \xi}\left(\frac{1}{\chi_{\mathrm{c}}+X}\right)\right\rangle w\right. \\
& \left.+\frac{1}{2}\left\langle\frac{1}{\chi_{\mathrm{c}}+X} \frac{\partial^{2}}{\partial \xi^{2}}\left(\frac{1}{\chi_{\mathrm{c}}+X}\right)\right\rangle w^{2}\right] \\
= & 2 g\left(s_{0}, \xi\right) \int_{0}^{\infty} \mathrm{e}^{-\chi_{\mathrm{c}} \cdot s}\left\langle\left[ 1+\frac{1}{2} s^{2} X^{\prime} w\right.\right. \\
& \left.\left.+\frac{1}{2}\left\{\frac{s^{4}}{4}\left(X^{\prime}\right)^{2}-\frac{s^{3}}{3} X^{\prime \prime}\right\} w^{2}\right] \mathrm{e}^{-X s}\right\rangle \mathrm{d} s
\end{aligned}
$$

where $\chi_{\mathrm{c}}=\chi_{\mathrm{c}}(\xi)$ is the continuous and $X=X(\xi)$ the line extinction coefficient (Eq. (1)). Derivatives with respect to $\xi$ are denoted by a prime, e.g. $X^{\prime}=\partial X(\xi) / \partial \xi$.

In the second part of Eq. (B.1) we have neglected the wavelength derivatives of the continuous extinction, i.e. assumed $\chi_{\mathrm{c}}(\xi)^{\prime}=0$ and $\chi_{\mathrm{c}}(\xi)^{\prime \prime}=0$ (cf. Sect. 2).

For Poisson distributed lines the coefficient of the "static" term in (B.1), $\Omega_{0}=\Omega(\xi, \xi, 0)$, is given by Eq. (11). The evaluation of the remaining coefficients of Eq. (B.1) requires the calculation of expectation values of the type $\left\langle\left[X^{(i)}\right]^{m}\left[X^{(j)}\right]^{n} V\right\rangle$ with $V=\exp (-X s)$ which can be achieved by using the identity (A.4). Thus far the method is not restricted to any specific stochastic distribution. Assuming now that the lines are Poisson distributed,

$$
\begin{aligned}
& \left\langle\exp \left(\lambda \cdot X^{(i)}+\mu \cdot X^{(j)}-X(\xi) s\right)\right\rangle= \\
& \quad \exp \left(\int_{\Theta} \int_{-\infty}^{\infty} \varrho(\hat{\xi}, \vartheta)\left\{\mathrm{e}^{h}-1\right\} \mathrm{d} \hat{\xi} \mathrm{d} \vartheta\right)
\end{aligned}
$$

holds with

$$
h \equiv h(\hat{\xi}, \vartheta, \xi-\hat{\xi} ; s)=\lambda \cdot \chi^{(i)}+\mu \cdot \chi^{(j)}-\chi \cdot s,
$$

where $\chi=\chi(\hat{\xi}, \vartheta, \xi-\hat{\xi})$ is the characteristic line profile (Eq. (7)) of the Poisson process. Here again $X^{(i)}$ and $\chi^{(i)}$ are the $i$ th partial derivatives of $X(\xi)$ and $\chi(\xi)$, respectively, and $X^{(0)}=X, X^{(1)}=$ $X^{\prime}, X^{(2)}=X^{\prime \prime}$. 
Note that in this Appendix we frequently drop the arguments of functions in order to gain a better overview.

Carrying out the differentiations of the expectation value (B.2) according to the identity (A.4), followed by setting $\lambda, \mu=0$, we obtain

$$
\begin{aligned}
\left\langle X^{\prime} \mathrm{e}^{-X s}\right\rangle & =\left.\frac{\partial}{\partial \lambda}\left\langle\mathrm{e}^{\lambda X^{\prime}} \cdot \mathrm{e}^{-X s}\right\rangle\right|_{\lambda=0} \\
& =\Omega_{0} \cdot \mathcal{E}\left(\chi^{\prime}\right), \\
\left\langle\left(X^{\prime}\right)^{2} \mathrm{e}^{-X s}\right\rangle & =\left.\frac{\partial^{2}}{\partial \lambda^{2}}\left\langle\mathrm{e}^{\lambda X^{\prime}} \cdot \mathrm{e}^{-X s}\right\rangle\right|_{\lambda=0} \\
& =\Omega_{0} \cdot\left[\left(\mathcal{E}\left(\chi^{\prime}\right)\right)^{2}+\mathcal{E}\left(\left(\chi^{\prime}\right)^{2}\right)\right], \\
\left\langle X^{\prime \prime} \mathrm{e}^{-X s}\right\rangle & =\left.\frac{\partial}{\partial \lambda}\left\langle\mathrm{e}^{\lambda X^{\prime \prime}} \cdot \mathrm{e}^{-X s}\right\rangle\right|_{\lambda=0} \\
& =\Omega_{0} \cdot \mathcal{E}\left(\chi^{\prime \prime}\right)
\end{aligned}
$$

where the abbreviation $\mathcal{E}(Y(\hat{\xi}, \vartheta, \xi-\hat{\xi}))$ is defined by Eq. (14). Note that $\Omega_{0}$ as well as $\mathcal{E}$ depend also on the variable $s$.

Comparison of the terms of the same power of $w$ provides the connection to the coefficients $\omega_{1}$ and $\omega_{2}$ obtained in Sect. 6 by the "direct" method.

In order to derive the coefficients $\tilde{\omega}_{1}$ and $\tilde{\omega}_{2}$ for the monochromatic radiative acceleration we need in addition

$\left\langle X X^{\prime} \mathrm{e}^{-X s}\right\rangle,\left\langle X\left(X^{\prime}\right)^{2} \mathrm{e}^{-X s}\right\rangle$, and $\left\langle X X^{\prime \prime} \mathrm{e}^{-X s}\right\rangle$ which can in principle be calculated in an analogous way as done for (B.4) to (B.6). However, in view of the result that the difference of the radiative acceleration from its static value is at most of the order of $w^{4}$ (Sects. 3, 6.3), we do not give further details here.

\section{References}

Baschek, B., Efimov, G. V., von Waldenfels, W., \& Wehrse, R. 1997, A\&A, 317, 630

Baschek, B., von Waldenfels, W., \& Wehrse, R. 2001, A\&A, 371, 1084

Blinnikov, S. I. 1996, Astron. Lett., 22, 79

Feller, W. 1966, An Introduction to Probability Theory and Its Applications, Vol. II (John Wiley \& Sons, New York)

Fisz, M. 1978, Wahrscheinlichkeitsrechnung und mathematische Statistik (VEB Deutscher Verlag der Wissenschaften, Berlin)

Karp, A. H., Lasher, G., Chan, K. L., \& Salpeter, E. E. 1977, ApJ, 214, 161

Learner, R. C. M. 1982, J. Phys. B, 15, L891

Pinto, P. A., \& Eastman, R. G. 2000, ApJ, 530, 757

Wehrse, R., Baschek, B., \& von Waldenfels, W. 2000a, A\&A, 359, 780, Paper I

Wehrse, R., Baschek, B., \& von Waldenfels, W. 2000b, A\&A, 359, 788, Paper II

Wehrse, R., von Waldenfels, W., \& Baschek, B. 1998, JQSRT, 60, 963 